\title{
Molecular Cloning, Characterization, and Expression Analysis of the CXCR4 Gene from Turbot: Scophthalmus maximus
}

\author{
Airong Jia and Xiao-Hua Zhang \\ Department of Marine Biology, Ocean University of China, 5 Yushan Road, Qingdao 266003, China \\ Correspondence should be addressed to Xiao-Hua Zhang, xhzhang@ouc.edu.cn \\ Received 16 February 2009; Revised 1 June 2009; Accepted 18 June 2009 \\ Recommended by Thomas Griffith
}

\begin{abstract}
Chemokine receptor 4 (CXCR4) belongs to the large superfamily of G protein-coupled receptors. The EST sequence of CXCR4 from turbot (Scophthalmus maximus L.) was obtained from a subtractive cDNA library. In the present study, the full-length cDNA sequence of turbot CXCR4 was obtained, and sequence analysis indicated that its primary structure was highly similar to CXCR4 from other vertebrates. Quantitative real-time PCR demonstrated that the highest expression level of turbot CXCR4 was in the spleen following injection with physiological saline (PS). After turbot were challenged with Vibrio harveyi, the lowest expression level of CXCR4 was detected at 8 hours in the spleen and 12 hours in the head kidney, and then increased gradually to 36 hours. These findings suggested that CXCR4 may play a significant role in the immune response of turbot.
\end{abstract}

Copyright ( 2009 A. Jia and X.-H. Zhang. This is an open access article distributed under the Creative Commons Attribution License, which permits unrestricted use, distribution, and reproduction in any medium, provided the original work is properly cited.

\section{Introduction}

Chemokines have been implicated in several immune mediated responses, such as inflammation, antigen presentation, blood cell development, viral infection, and wound healing [1-3]. The specific effects of chemokines are mediated through a subset of the G-protein coupled receptors [4]. Most of these receptors have been reported to interact with multiple ligands, and most of ligands interact with more than one receptor [5]. A notable exception is the stromal cellderived factor-1 (SDF-1, also called CXCL12)/chemokine receptor 4 (CXCR4) interaction [6, 7]. Recently, a new receptor, CXCR7, was reported as an alternative nonsignaling SDF-1 receptor, suggesting that the CXCR4/SDF1 relationship is not entirely exclusive. However, CXCR7, unlike CXCR4, is only expressed in limited tissues, and its role is not quite clear [8]. SDF-1 belongs to the non-ELR subgroup of CXC chemokines and has a role to attract lymphocytes and monocytes, with poor chemotactic ability for neutrophils [9]. This ligand interacts specifically with CXCR4, which is one of the best studied chemokine receptors primarily due to its role as a target in the entry of $\mathrm{T}$ cell-tropic HIV $[7,10]$ as well as the ability to mediate the metastasis of some cancers [6].
CXCR4 was initially thought to be a membrane protein. However, immunohistochemical results of CXCR4 in breast cancer tissues showed that its subcellular localization could vary, for example, on the membrane, in the cytoplasm, or even in the nucleus. Based on these findings, CXCR4 could serve as a novel biomarker for cancer metastasis and even the inflammatory reaction [11]. CXCR4/SDF-1 interaction is necessary in the immune response $[3,12]$, and CXCR4 influences the immune system under physiologic and pathologic conditions through negative regulation of MHC class II expression [13].

In order to clarify the role of CXCR4 in disease, a fundamental understanding of the factors regulating expression is critical. A number of signaling molecules have been shown to affect CXCR4 transcription. For example, its expression may be increased as a result of intracellular second messengers and cytokine growth factors. On the other hand, inflammatory cytokines have been shown to attenuate CXCR4 expression [14]. Of additional interest are those factors that regulate CXCR4 expression and affect disease progression [15].

In our previous study, suppression subtractive hybridization $(\mathrm{SSH})$ was used to investigate the response of turbot to Vibrio harveyi using a cDNA library constructed from kidney 
and spleen of experimentally infected turbot, and several immune-related genes were identified, including a CXCR4 (D1B11) [16]. Some studies focused on the regulation of chemokines in response to bacterial infection and vaccination, since SDF-1 is thought to play an important role in the first line of defense against pathogens in fish [12, 17]. However, few studies have been performed in fish concerning the expression of its receptor. Based on the known role of CXCR4 and its ligand SDF-1 in homing of hematopoietic cells, CXCR4 is likely to play a role in metastasis $[6,7]$. We initiated a study aimed at dissecting additional functions of turbot CXCR4 in relation to the immune system.

\section{Materials and Methods}

2.1. Turbot. Apparently healthy turbot (length $=13 \pm 1 \mathrm{~cm}$, mass $=45 \pm 2 \mathrm{~g}$ ) were purchased from Zhuoyue fish farm (Jiaonan, Shandong Province, China), and acclimated to laboratory conditions for 1 week in aerated static seawater at $16-20{ }^{\circ} \mathrm{C}$.

2.2. Primer Design. According to the EST sequence of CXCR4, which was obtained from the turbot subtractive cDNA library in a previous study [16], two specific primers (CXCRGSP1 and CXCRGSP2) were designed in order to carry out $5^{\prime}$ - and $3^{\prime}$-RACE. CXCRGSP1 was used for the amplification of the $5^{\prime}$-end, and CXCRGSP2 was designed for the $3^{\prime}$-end. The universal primer (UPM) used for $5^{\prime}$ - and $3^{\prime}$-RACE was the mixture of the long and short primer (from SMART RACE cDNA Amplification Kit, Clontech). A pair of primers, RTCXCRS and RTCXCRA, was designed according to the full-length cDNA sequence and used to amplify a cDNA fragment of 117 bp from turbot tissue cDNA samples for expression analysis. Details of the primers are listed in Table 1.

2.3. Isolation of RNA and Amplification of Full-Length cDNA. Total RNA was extracted with Trizol reagent (Invitrogen) from the spleen of turbot according to the manufacturer's protocol. To obtain full-length $5^{\prime}$ - and $3^{\prime}$-termini of the CXCR4 gene, the SMART RACE cDNA Amplification Kit (Clontech) was used [16].

2.4. Sequence Analysis. The data of DNA sequences were edited and analyzed using DNASTAR 5.0, and the similarity of all sequences were analyzed by BLASTN and BLASTP at the National Center of Biotechnology Information [18]. For transmembrane domains, the TMHMM Server 2.0 program was used (http://www.cbs.dtu.dk/services/TMHMM-2.0/). The primary structure was analyzed by ProtParam (http://cn.expasy.org/tools/protparam.html), and the secondary structure was predicted by PHD program (http://www.predictprotein.org/).

2.5. Sequence Alignment. The sequences used for alignment were retrieved using BLASTN. Multiple alignments of the amino-acid sequences were obtained by the software ClustalX1.81. A phylogenetic tree was performed using
MEGA3.1 by NJ (Neighbor-Joining) method. Reliability of the NJ tree was assessed by the interior branch test, using 1000 replications.

2.6. Preparation of $V$. harveyi. $V$. harveyi VIB 645 was obtained from the School of Life Sciences, Heriot-Watt University, UK, and was previously confirmed to be very pathogenic to fish [19]. It was cultured at $28 \circ \mathrm{C}$ on marine $2216 \mathrm{E}$ agar plates and harvested in the logarithmic phase of growth, after $\sim 12$ hours. The cell numbers were calculated by the method of Plate Count (PC) [20]. In brief, the bacterial suspension was serially 10 -fold diluted with sterile physiological saline, and each dilution was plated on triplicate plates of $2216 \mathrm{E}$ agar for calculating the colonies. The bacteria were then suspended in physiological saline (PS)

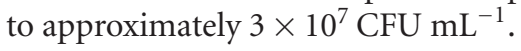

2.7. Challenge and Sampling. The bacterial suspension was injected intraperitoneally in $0.15 \mathrm{~mL}$ volumes into a group of 35 turbot (the injection dose is around the $\mathrm{LD}_{50}$ values, which is $\left.1.4 \times 10^{5} \mathrm{CFU} \cdot \mathrm{g}^{-1}\right)$. In parallel, a group of 4 fish was injected with PS as controls, and another group of 4 fish was noninjected as blank controls. Subgroups of 4 bacterial-infected fish were sacrificed at 4, 8, 12, 24 and 36 hours. The controls were killed after 8 hours. The remaining fish died successively after infection and were not used further. Samples of head kidney, kidney, heart, liver, intestine, muscle, spleen, and gill were collected and kept at $-80^{\circ} \mathrm{C}$.

2.8. Quantitative Real-Time PCR. The tissues from each subgroup of four turbot were pooled, and total RNA were extracted. DNA contamination was removed by DNase I (Takara) treatment, and the purity was verified by PCR amplification of $\beta$-actin mRNA using $\beta$-actin gene specific primers (RTactinS and RTactinA, Table 1). The cDNA was generated with M-MLV reverse transcriptase (Promega). A total of $2 \mu \mathrm{g}$ RNA from each kind of tissue was reverse transcribed in a final volume of $25 \mu \mathrm{L}$ at $42^{\circ} \mathrm{C}$ for 60 minutes. Finally, cDNA was diluted to 1: 4 with sterile water, and stored at $-20{ }^{\circ} \mathrm{C}$ until use. Real-time PCR was performed as described previously [16]. Turbot $\beta$-actin was used as a control to normalize the starting quantity of RNA $[3,21]$, and a fragment of $108 \mathrm{bp}$ was amplified using the primers RTactinS and RTactinA (Table 1). All samples were amplified in triplicate.

2.9. Statistics of Quantitative Real-Time PCR. The $\beta$-actin of each reaction was used to normalize the level of total RNA. Statistical analysis was performed with SPSS13.0 software. Significant differences between samples were analyzed via one-way ANOVA (analysis of variance) using Duncan's test [22]. Differences of $p<.05$ were considered significant.

\section{Results}

3.1. Cloning and Characteristics of CXCR4 Gene from Turbot. The EST sequence of CXCR4, which had a sequence of 
TABle 1: Primer sequences used in this study.

\begin{tabular}{ll}
\hline Primer ID & Primer sequences \\
\hline Long primer & $5^{\prime}$-CTAATACGACTCACTATAGGGCAAGCAGTGGTATCAACGCAGAGT-3' \\
Short primer & $5^{\prime}$-CTAATACGACTCACTATAGGGC-3' \\
CXCRGSP1 & $5^{\prime}$-CACAGTTAGCAGGGCGGCAGGC-3' \\
CXCRGSP2 & $5^{\prime}$-TGCACATGATCTACACGGTCAACCTG-3' \\
RTCXCRS & $5^{\prime}$-ATCATTGGCAACGGATTAGTGGTG-3' \\
RTCXCRA & $5^{\prime}$-CAGCGTGAGGACGAACAGGAGG-3' \\
RTactinS & $5^{\prime}$-TGAACCCCAAAGCCAACAGG-3' \\
RTactinA & $5^{\prime}$-CAGAGGCATACAGGGACAGCAC-3' \\
\hline
\end{tabular}

56 bp with unknown $5^{\prime}$ - and $3^{\prime}$-ends, was obtained from the subtractive cDNA library after turbot were injected with $V$. harveyi [15]. The full-length cDNA sequence of CXCR4 (GenBank accession number: EF373652) was obtained by the methods of $5^{\prime}$ - and $3^{\prime}$-RACE. The full-length cDNA contained a 112 bp $5^{\prime}$-UTR, a 1119 bp open reading frame (ORF) encoding a polypeptide of 372 amino-acid residues, and a $116 \mathrm{bp} 3^{\prime}$-UTR. The $3^{\prime}$-UTR contains a single typical polyadenylation signal (AATAAA) from nucleotide 1341 to 1346. Based upon the amino-acid sequences of CXCR4 from other organisms and the analysis of TMHMM Server 2.0, the predicted protein had seven transmembrane domains (TMs) (Figure 1).

According to the predication by ProtParam program, turbot CXCR4 had a molecular mass of $41.7 \mathrm{kDa}$ and theoretical pI of 8.94. The total number of negatively (Asp + Glu) and positively (Arg + Lys) charged residues was 25 and 34, respectively. The instability index (II) of CXCR4 was computed to be 35.43 , and so it was classified as a stable protein.

Turbot CXCR4 had two N-glycosylation sites (N $\left[^{\wedge} \mathrm{P}\right][\mathrm{ST}]$ $[\wedge \mathrm{P}])$ located at 12 and 16 aa. Six protein kinase $\mathrm{C}$ phosphorylation sites $([\mathrm{ST}][\mathrm{RK}])$ were located at $77,151,332,340$, 344, and 351 aa; six casein kinase II phosphorylation sites ([ST].\{2\}[DE]) were at 75, 85, 172, 292, 305, and 362 aa; three N-myristoylation sites (G ${ }^{\wedge}$ EDRKHPFYW $] .\{2\}$

[STAGCN][^P]) were at 56,109, and 355 aa. At the site of 126 aa, there was a G-protein coupled receptors signature [GSTALIVMFYWC][GSTANCPDE][^EDPKRH].\{2\} [LIVMNQGA] $\{2\}$ [LIVMFT][GSTANC][LIVMFYWSTAC] [DENH]R[FYWCSH].\{2\}[LIVM]. The extracellular regions of the turbot CXCR4 contained four cysteines, presumably forming disulfide bonds.

According to the calculation of PHD, turbot CXCR4 was classified as a mixed protein that contained $48.66 \%$ alpha helix, $7.53 \%$ beta pleated sheet, and $43.82 \%$ aperiodical coil.

3.2. Phylogenetic Analysis and Alignment. A condensed phylogenetic tree was constructed based on the amino-acid sequences of CXCR4 in different organisms (Figure 2). The overall topology of the tree showed that the turbot CXCR4 was most similar to CXCR4 from medaka (Oryzias latipes T.) and also had high similarity with those from other organisms, especially from fish. The CXCR6 in rat (Rattus norvegicus $\mathrm{B}$.) formed a distinct paraphyletic cluster.
Alignment of amino-acid residues of the turbot CXCR4 with those from other vertebrates indicated high level of amino-acid sequence conservation (Figure 1). The protein showed $69 \%-72 \%$ identity with those of other fish. The percent identity with the other vertebrate CXCR4 varied in the $61-63 \%$ range. The seven transmembrane domains were highly conserved in all organisms.

3.3. Expression of CXCR4 mRNA in Different Tissues of Turbot. Real-time PCR was conducted to analyze the tissue expression of turbot CXCR4. The data showed high variation among different tissues with a fold change up to 300 . There were high variations between PS-injected and blank control turbot in head kidney, kidney, and spleen expressions (Figure 3). Based on the fold changes relative to heart, the CXCR4 mRNA was most abundant by 268 -fold in the spleen of PS-injected turbot. The expression level of CXCR4 in head kidney, kidney, and gill was approximately 100 -fold, and in liver was 22 -fold higher than that in heart. There was no significant difference among the expression levels in muscle, intestine, and heart (Figure 3 ).

Fifteen fish died from 20 to 36 hours after injection of $V$. harveyi, so 20 survivors were used in the expression analysis. The expression level of CXCR4 was analyzed in head kidney and spleen in which the expression levels fluctuated after injection (Figure 4).

In the head kidney, the expression level of CXCR4 in PSinjected turbot reduced approximately 4.5 -fold relative to the noninjected fish. Comparing with the noninjected samples, the expression level of CXCR4 decreased initially, and started to increase from 24 hours (Figure 4(a)). In the spleen, the expression level decreased at 4 hours and was lowest, that is, 9-fold lower than PS-injected turbot, at 8 hours before increasing rapidly at 12 hours, and then returned to the background level at 36 hours. The expression level of CXCR4 in PS-injected fish was much higher than that in noninjected fish $(p<.001)$ (Figure $4(\mathrm{~b}))$

\section{Discussion}

The immune system of fish is very different from mammals. Thus in fish, the innate immune system regarded as a fundamental defense mechanism [23]. In particular, fish lack bone marrow and lymph nodes; instead they use kidney as a major lymphoid organ [3]. Besides, fish have splenic immune 
SmCXCR4
OlCXCR4
DrCXCR4
CcCXCR4
ArCXCR4
GgCXCR4
RnCXCR4
MmCXCR4
HsCXCR4
Clustal Consensu

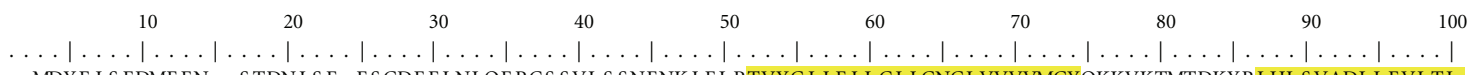
- -MDYE I S FDMF EN - - STDNI SE - ES GDF E LNLQE PCS SVL S SNFNK I F L PTVYGI I F I L GI I GNGLVVVVMGYQKKVKTMTDKYRLHL SVADL L FVL TL - -MEY FYES I VFDN - - S S EG I LD - GS GDF E F - PEEAYKEAL SRDF KK I F L P TVYGV I FV L GI VGNGLVVVVMGYQKKVKNMTDKYR LHL SVADL L LVLTL - - MAY - YGHI VF EDDL S ADNS S E F GS GD I GANF EVPCDVEVS HDFQR I F L P TVYG I I FA L GL I GNGLVVL VMGCQKKS RTMTDKY R LHL S VADL L FVL T L - -ME F - YDHI F FD - - - - - NS SDS GS GDF - - DFDE LCDL KVSNDFQK I F L PVVYG I I FV L GI I GNGLVVLVMGFQKKS KNMTDKYR LHL S I ADL L FVL TL MDYET - - - - -WTVDFT F ENNT EGS GS GDY S - QYDEVCKRNLNGDL RK I F L PTVYT I I FVMGI VGNGLVV I VMGYQK - VKTMTDKYR LHL L I ADL L FVF T L MDGLDL S S G - - I L I E FADNGS E E I GS ADYG - DYGE PCFQHENADFNR I F L P T I Y S I I F L T GI I GNGLV I I VMGYQKKQR SMTDKYR LHL SVADL L FV I TL - . . . . - . - ME I Y T SDNY S EEVGS GDYD - SNKE PCF RDENENFNR I F L PT I Y F I I F L T GI VGNGLV I LVMGYQKKLRSMTDKYRLHL SVADL L FV I TL ME P I S - - - - - VS I Y T SDNY S EEVGSGDYD - SNKE PCFRDENVHFNR I F L P T I Y F I I F L T GI VGNGLV I LVMGYQKKLR SMTDKYRLHL SVADL L FV I TL MEG I S S I P L P L LQ I Y T SDNYT E EMGS GDYD - SMKE PCF RE ENANFNK I F L PT I Y S I I F L T GI VGNGLV I LVMGYQKKL R SMTDKYRLHL S VADL L FV I T L Clust Consensus

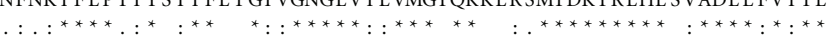
$110 \quad 120 \quad 130 \quad 140 \quad 150 \quad \ldots|\ldots| \ldots|\ldots| \ldots|\ldots| \ldots|\ldots| \ldots|\ldots| \ldots|\ldots| \ldots|\ldots| \ldots|\ldots| \ldots|\ldots| \ldots|\ldots| \ldots \mid$

SmCXCR4 OlCXCR4

DrCXCR4

CcCXCR4

ArCXCR4

GgCXCR4 RnCXCR4

MmCXCR4

HsCXCR4

Clustal Consensu

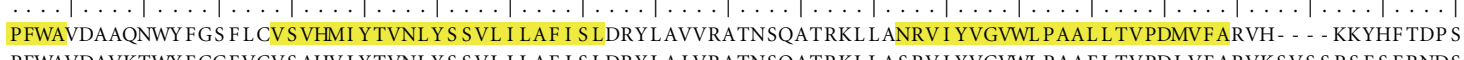
PFWAVDAVKTWY F GGFVCV S AHV I YTVNLY S SVL I LAF I S LDRY LA I VRATNSQATRKL LA SRV I YVGVWL PAAF LTVPDLVF ARVKS VS S P S F S F RNDS PFWAVDVAKDWY FGGFMCVAVHMI Y TVNLY S SVL I LAF I S LDRY LAVVRATNSQGPRKL LANR I I YVGVWL PAAL LTVPDL VF AKAES - . - . - . - . - . PFWAVDAA S GWHF GGF LCVTVNMI YTLNLY S SVL I LAF I S LDRY LAVVRATNSQNFRRVLAEKV I YLGVWL PAS L LTVPDL VFAKVHD - - - . - . - . - PFWAVDAAS SWY F GGF LCK I VNS I YTVNLY S SVL I LAF I S FDRY LAVVRATNSHKPRKL LAEK I I YVGVWL PAT L L TVPDLVFAQVHDEG - - . - . - . - . PFWS VDAA I SWY F GNVLCKAVHV I YTVNLY S SVL I LAF I S LDRY LA I VHATNSQR PRKL LAEK I VYVGVWL PAVL LTVPD I I FAS TS EV - - . - . - . - . PFWAVDAMADWY FGKF LCKAVHI I YTVNLY S SVL I LAF I S LDRY LA I VHATNSQS ARKL LAEKAVYVGVWI PAL LL T I PD I I FADVSQG - - - - - - - - - - PFWAVDAMADWY FGKF LCKAVHI I YTVNLY S SVL I LAF I S LDRY LA I VHATNSQR PRKL LAEKAVYVGVWI PALLLT I PDF I FADVSQGD - . - . - . - . PFWAVDAVANWY F GNF LCKAVHV I YTVNLY S S VL I LAF I S LDRY LA I VHATNSQR PRKL LAEKVVYVGVWI PALLLT I PDF I FANVS EA - - . - . - . - - -

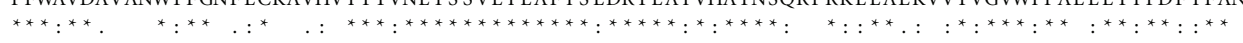

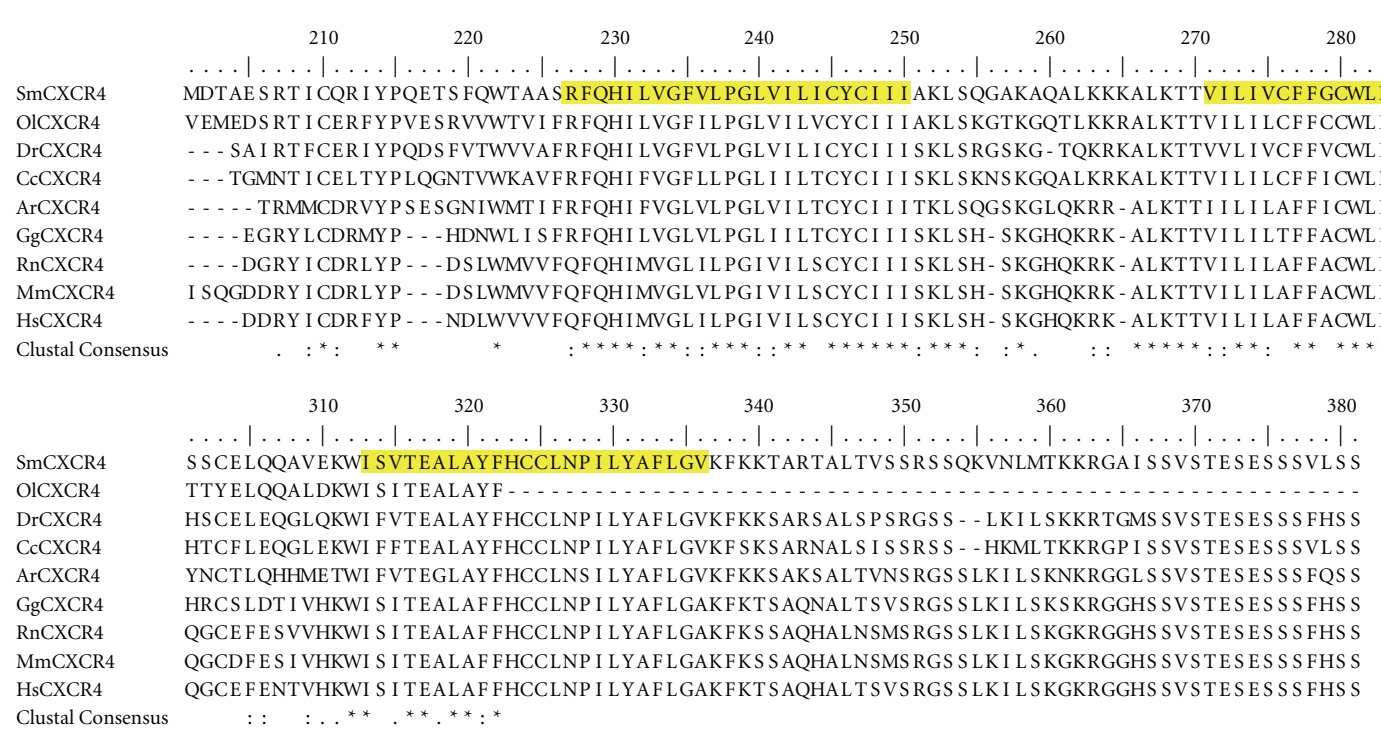

Figure 1: Alignment of deduced amino-acid sequences of turbot CXCR4 with others via ClustalX 1.8. Receptor designations: Sm-S. maximus, Ol-O. latipes, Dr-Danio rerio, Cc-Cyprinus carpio, Ar-Acipenser ruthenus, Gg—Gallus gallus, $\mathrm{Rn}-\mathrm{R}$. norvegicus, $\mathrm{Mm}-$ Mus musculus, Hs-Homo sapiens. Symbols: $*$, identical residues in all sequences; :, conserved substitutions; ., semiconserved substitutions; -, gaps introduced during the alignment process. Seven transmembrane domains of CXCR4 are marked.

function that centers on lymphocytes, macrophages, and many kinds of granulocytes [24]. In order to obtain RNA representatives of immune systems, head kidney and spleen tissues of turbot were used to construct the subtractive cDNA library by the SSH method in the previous study. This revealed that several immune-related genes were identified that should be useful for analyzing gene function during disease defence and for developing molecular markers related to disease resistance.

In fish, the CXCR4 gene has also been identified in several other species such as rainbow trout [25], carp [26] and sterlet [27]. In this study, the full-length cDNA of turbot CXCR4 was obtained for the first time, encoding a peptide of 372 aa. The protein had two N-glycosylation sites that were important for SDF binding, and the extracellular cysteines forming disulfide bonds stabilized the structure of this protein. CXCR4 was structurally divided into 15 domains: seven transmembrane, four intracellular, and four extracellular domains. Each domain was well-conserved among those CXCR4 counterparts in various animal species, especially the intracellular domains and the seven transmembrane domains (Figure 1). The CXCR4 sequences from the phylogenetically diverged lineages were compared with the sequences of the other chemokine receptors to determine the CXCR4-specific structural elements. Thus, it was reasonable to suggest that these elements confer selectivity on CXCR4 ligand binding and signaling.

From the present study, the high-level elevation of CXCR4expression occurred in spleen, kidney and gills, which corresponded well to the major roles of these three 


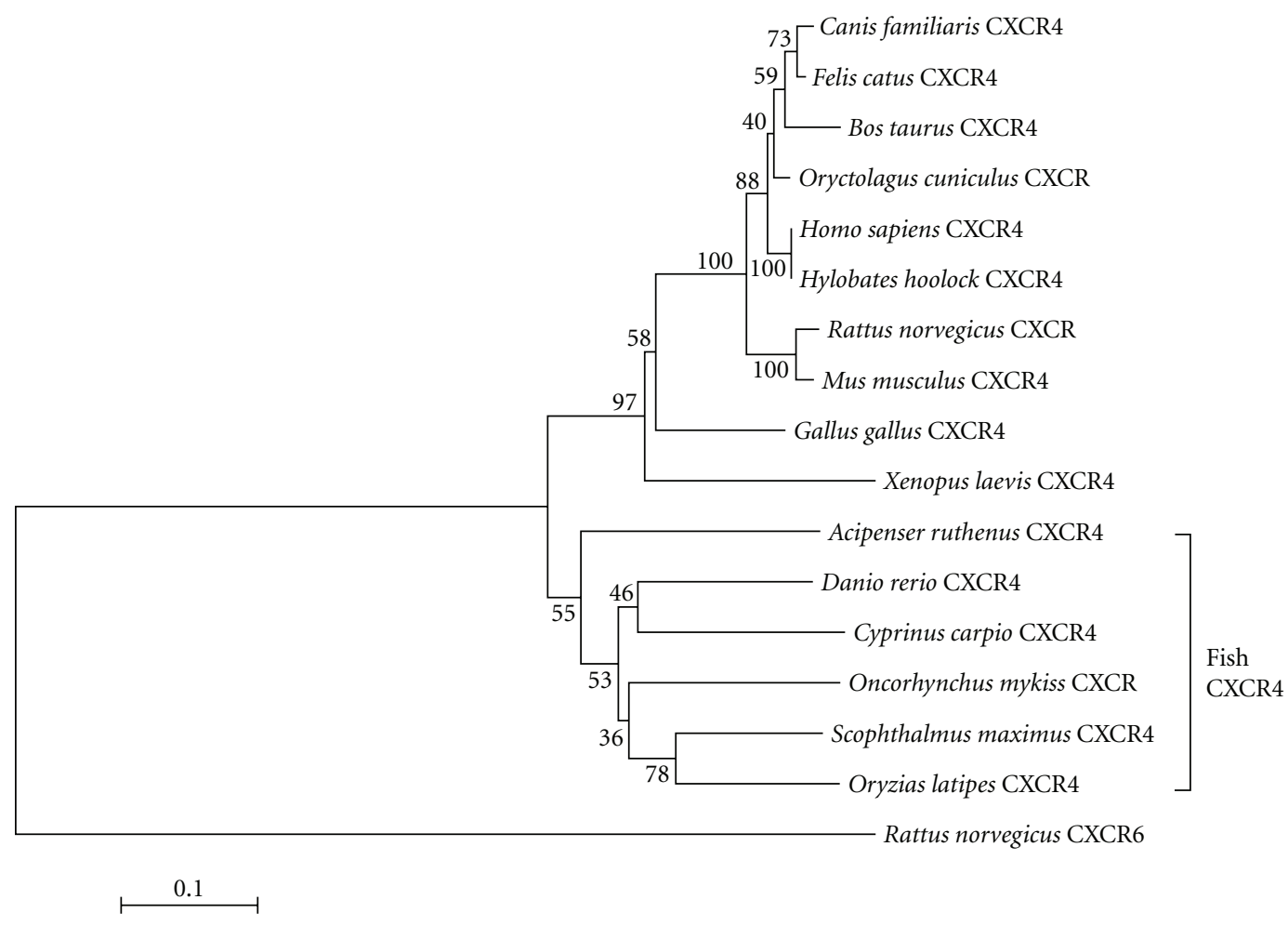

FIGURE 2: Neighbor-Joining tree of the amino-acid sequences of CXCR4. Numbers at tree nodes refer to percent bootstrap values after 1000 replicates; the scale bar refers to a phylogenetic distance of 0.1 amino acid substitutions per site. CXCR6 of Rattus norvegicus was used as outgroup. Turbot CXCR4 is underlined. The GenBank accession numbers of the sequences are as follows, CXCR4: D. rerio, AAH50172; $C$. carpio, BAA32797; O. latipes, ABC41565; A. ruthenus, CAB60252; H. sapiens, CAA12166; Hylobates hoolock, AAF89348; Canis familiaris, ABA28309; Bos Taurus, AAI05218; G. gallus, AAG09054; M. musculus, BAA19187; Xenopus laevis, AAI10722. CXCR: Oncorhynchus mykiss, CAA04493; Oryctolagus cuniculus, ABX55954; Felis catus, CAA08839; R. norvegicus, AAB50408. CXCR6: R. norvegicus, AAZ66333.

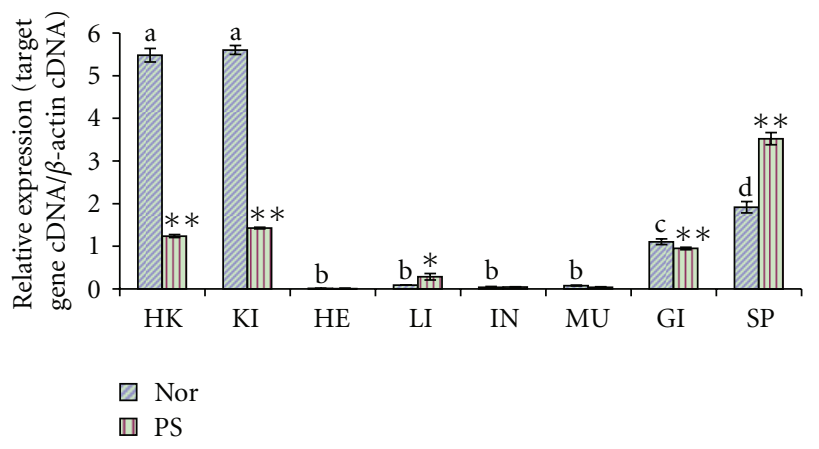

FIGURE 3: Quantitative analyses of the expression profiles CXCR4 gene in different tissues: head kidney (HK), kidney (KI), heart (HE), liver (LI), intestine (IN), muscle (MU), gill (GI) and, spleen (SP). Tissues were harvested from the PS-injected (PS) and noninjected (Nor) turbot. The expression level was analyzed by one-way ANOVA followed by Duncan's test. Groups marked with the same letters are not statistically different. ${ }^{*} p<.05 ;{ }^{* *} p<.001$ as compared to the control.

tissues played in fish immune system [12, 28, 29]. It might suggest that the CXCR4 was relative to the immune system and it had a high constitutive expression in head kidney, kidney and spleen. CXCR4 was also constitutively expressed in canine although at different levels [17]. When turbot were injected with PS, the CXCR4 expression levels in head kidney and kidney were reduced compared with those of noninjected turbot. Nevertheless, the expression of CXCR4 was induced in the spleen. This result may be related to the inflammatory responses. Although stimulusinduced proinflammatory molecules such as interleukin or tumor necrosis factor $(\mathrm{TNF} \alpha)$ are important in avoiding the growth and dissemination of gram-negative bacteria, their overproduction can lead to endotoxin shock which is a severe systemic inflammatory response, characterized by fever, myocardial dysfunction, acute respiratory failure, hypotension, multiple organ failure, and in a large number of cases, death [30, 31]. Previous studies have shown that there is a tight control of CXCR4 through negative regulation of immune factor to avoid an excess of inflammation $[13,30]$. In addition, a high level of SDF-1 can induce the internalization and degradation of CXCR4 through the lysosome pathway $[32,33]$. This suggested that the injection might change the microenvironment of turbot and decrease the expression of CXCR4, which could cause the inflammatory response in the head kidney. It is previously reported that the cytokine of TNF was induced at 8 hours after PBS-injected in turbot kidney [34], and the SDF-1 may be induced for the participation of inflammatory reaction and the degradation of CXCR4. The reason for the induced expression of CXCR4 


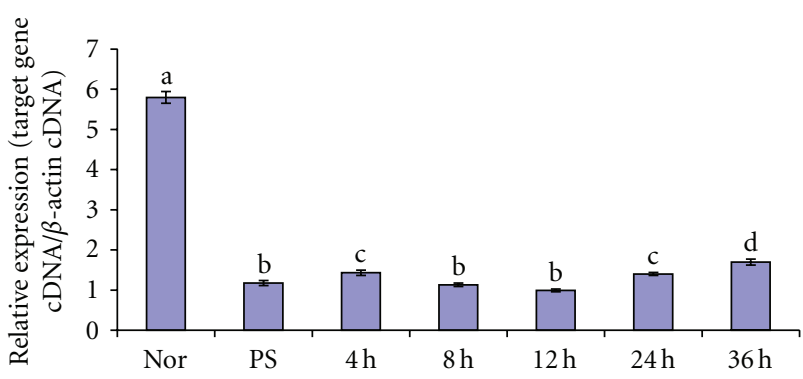

(a)

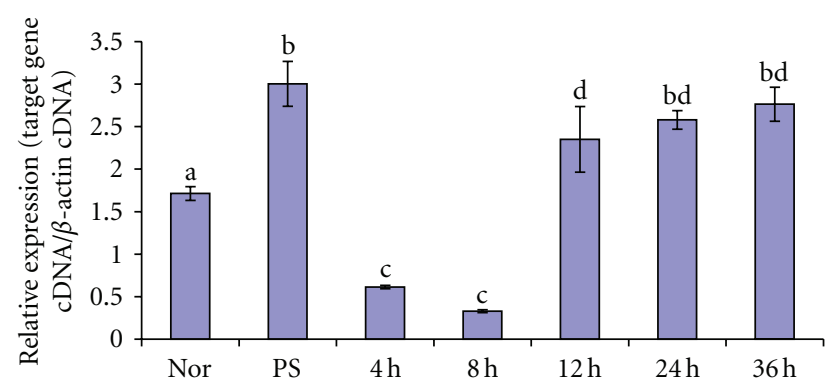

(b)

FIgURE 4: Expression profiles of the CXCR4 gene in different tissues of turbot using real-time PCR at different times after challenged with $V$. harveyi (4, 8, 12, 24 and 36 hours). (a) head kidney; (b) spleen. Nor: noninjected fish; PS: PS-injected fish. The expression level was analyzed by one-way ANOVA followed by Duncan's test. Groups marked with the same letters are not statistically different.

at 8 hours in the spleen is thought to control an excess of inflammatory response and protect the host from the endotoxic or septic shock, although the involved mechanisms are not fully understood. The response may be also correlated with reduced SDF-1 expression, and this finding agreed with previous study, in which the expressions of all nine immune-related genes (ISG15, SIC, IRF1, IRF7, IRF10, MHC I, viperin, LGP2, and TLR3) were suppressed in the 6 hours saline control spleen samples relative to 0 hour controls [35].

After turbot were challenged with $V$. harveyi, the expression level of CXCR4 was decreased both in spleen and head kidney, and then increased gradually. The downregulation of chemokine receptors by pathogens is a common pathogenic effect [36]. For example, viral hemorrhagic septicemia virus (VHSV) infection in rainbow trout induced a downmodulation of the levels of transcription of IL-8 receptor early after infection in spleen and head kidney [37]. Previous studies found that the high levels of SDF-1 could reduce the expression of CXCR4 [32, 33]. In peripheral blood mononuclear cells (PBMC) of canine or human, while CXCR4 mRNA was expressed at a higher level, the expression of SDF-1 mRNA was hardly detected [17, 38, 39]. Therefore, the data for turbot CXCR4 obtained here matched to those of the expression of SDF-1. In the large yellow croaker, the expression of SDF-1 was induced initially and then decreased in kidney and spleen postinduction by bacterial vaccine [12]. This expression pattern was mostly matched to the expression of turbot CXCR4 in head kidney and spleen, in which the expression levels were suppressed at the very beginning prior to increasing. These results suggest that turbot might have experienced an evolutionary selective pressure to avoid excessive inflammatory states which is associated with an increased activity of the CXCR4. However, another study found that SDF-1 chemokine was not induced in catfish under bacterial challenge with Edwardsiella ictaluri [9]. A possible explanation is that a functional differentiation might occur among SDF-1 chemokines from different fish species. Moreover, different experimental conditions used in the studies might also result in variations in expression pattern. Therefore, the elucidation of the turbot SDF-1 is essential for understanding the biological activity of CXCR4 and possible for clearing that if SDF-1 could be negative regulated by CXCR4 in turbot. However, many genetic approaches (e.g., gene knockout) are not available in turbot, which causes some difficulties in studying the interaction between CXCR4 and SDF-1 [40,41].

\section{Acknowledgments}

This work was supported by a Grant from the NSFC-RGC Joint Research Scheme (no. 30831160512), a Grant from the National Natural Science Foundation of China (no. 30771656), and the program for New Century Excellent Talents in University (no. NCET-04-0645).

\section{References}

[1] J. C. Cyster, "Chemokines and cell migration in secondary lymphoid organs," Science, vol. 286, no. 5447, pp. 2098-2102, 1999.

[2] D. Rossi and A. Zlotnik, "The biology of chemokines and their receptors," Annual Review of Immunology, vol. 18, pp. 217243, 2000.

[3] Y. Liu, S.-L. Chen, L. Meng, and Y.-X. Zhang, "Cloning, characterization and expression analysis of a CXCL10-like chemokine from turbot (Scophthalmus maximus)," Aquaculture, vol. 272, no. 1-4, pp. 199-207, 2007.

[4] P. M. Murphy, M. Baggiolini, I. F. Charo, et al., "International union of pharmacology. XXII. Nomenclature for chemokine receptors," Pharmacological Reviews, vol. 52, no. 1, pp. 145$176,2000$.

[5] A. Mantovani, "The chemokine system: redundancy for robust outputs," Immunology Today, vol. 20, no. 6, pp. 254-257, 1999.

[6] Q. Ma, D. Jones, P. R. Borghesani, et al., "Impaired Blymphopoiesis, myelopoiesis, and derailed cerebellar neuron migration in CXCR4- and SDF-1-deficient mice," Proceedings of the National Academy of Sciences of the United States of America, vol. 95, no. 16, pp. 9448-9453, 1998.

[7] T. Nagasawa, K. Tachibana, and T. Kishimoto, "A novel CXC chemokine PBSF/SDF-1 and its receptor CXCR4: their functions in development, hematopoiesis and HIV infection," Seminars in Immunology, vol. 10, no. 3, pp. 179-185, 1998.

[8] H. Shim, S. Oishi, and N. Fujii, "Chemokine receptor CXCR4 as a therapeutic target for neuroectodermal tumors," Seminars in Cancer Biology, vol. 19, no. 2, pp. 123-134, 2009.

[9] P. Baoprasertkul, C. He, E. Peatman, S. Zhang, P. Li, and Z. Liu, "Constitutive expression of three novel catfish CXC chemokines: homeostatic chemokines in teleost fish," Molecular Immunology, vol. 42, no. 11, pp. 1355-1366, 2005. 
[10] S. Basmaciogullari, B. Pacheco, S. Bour, and J. Sodroski, "Specific interaction of CXCR4 with CD4 and CD8 $\alpha$ : functional analysis of the CD4/CXCR4 interaction in the context of HIV-1 envelope glycoprotein-mediated membrane fusion," Virology, vol. 353, no. 1, pp. 52-67, 2006.

[11] B. C. Schmid, M. Rudas, G. A. Rezniczek, S. Leodolter, and R. Zeillinger, "CXCR4 is expressed in ductal carcinoma in situ of the breast and in atypical ductal hyperplasia," Breast Cancer Research and Treatment, vol. 84, no. 3, pp. 247-250, 2004.

[12] X. Wan and X. Chen, "Molecular cloning and expression analysis of a CXC chemokine gene from large yellow croaker Pseudosciaena crocea," Veterinary Immunology and Immunopathology, vol. 127, no. 1-2, pp. 156-161, 2009.

[13] C. Sheridan, M. Sadaria, P. Bhat-Nakshatri, et al., "Negative regulation of MHC class II gene expression by CXCR4," Experimental Hematology, vol. 34, no. 8, pp. 1085-1092, 2006.

[14] S. K. Gupta, P. G. Lysko, K. Pillarisetti, E. Ohlstein, and J. M. Stadel, "Chemokine receptors in human endothelial cells. Functional expression of CXCR4 and its transcriptional regulation by inflammatory cytokines," Journal of Biological Chemistry, vol. 273, no. 7, pp. 4282-4287, 1998.

[15] J. M. Busillo and J. L. Benovic, "Regulation of CXCR4 signaling," Biochimica et Biophysica Acta, vol. 1768, no. 4, pp. 952-963, 2007.

[16] C. Wang, X.-H. Zhang, A. Jia, J. Chen, and B. Austin, "Identification of immune-related genes from kidney and spleen of turbot, Psetta maxima (L.), by suppression subtractive hybridization following challenge with Vibrio harveyi," Journal of Fish Diseases, vol. 31, no. 7, pp. 505-514, 2008.

[17] S. Tsuchida, A. Kagi, and T. Takahashi, "Characterization of cDNA and genomic sequences encoding a canine chemokine receptor, CXCR4 and its ligand CXCL12," Veterinary Immunology and Immunopathology, vol. 116, no. 3-4, pp. 219-225, 2007.

[18] S. F. Altschul, T. L. Madden, A. A. Schäffer, et al., "Gapped BLAST and PSI-BLAST: a new generation of protein database search programs," Nucleic Acids Research, vol. 25, no. 17, pp. 3389-3402, 1997.

[19] X.-H. Zhang and B. Austin, "Pathogenicity of Vibrio harveyi to salmonids," Journal of Fish Diseases, vol. 23, no. 2, pp. 93-102, 2000.

[20] X.-H. Zhang, Marine Microbiology, China Ocean University Press, Qingdao, China, 2007.

[21] Q. Zhong, Q. Zhang, Z. Wang, et al., "Expression profiling and validation of potential reference genes during Paralichthys olivaceus embryogenesis," Marine Biotechnology, vol. 10, no. 3, pp. 310-318, 2008.

[22] M. R. Stoline, "The status of multiple comparisons: simultaneous estimation of all pairwise comparisons in one-way ANOVA designs," The American Statistician, vol. 35, no. 3, pp. 134-141, 1981.

[23] A. E. Ellis, "Innate host defense mechanisms of fish against viruses and bacteria," Developmental and Comparative Immunology, vol. 25, no. 8-9, pp. 827-839, 2001.

[24] C. M. L. Press and O. Evensen, "The morphology of the immune system in teleost fishes," Fish and Shellfish Immunology, vol. 9, no. 4, pp. 309-318, 1999.

[25] G. D. Daniels, J. Zou, J. Charlemagne, S. Partula, C. Cunningham, and C. J. Secombes, "Cloning of two chemokine receptor homologs (CXC-R4 and CC-R7) in rainbow trout Oncorhynchus mykiss," Journal of Leukocyte Biology, vol. 65, no. 5, pp. 684-690, 1999.
[26] K. Fujiki, D.-H. Shin, M. Nakao, and T. Yano, "Molecular cloning of carp (Cyprinus carpio) CC chemokine, CXC chemokine receptors, allograft inflammatory factor-1, and natural killer cell enhancing factor by use of suppression subtractive hybridization," Immunogenetics, vol. 49, no. 10, pp. 909-914, 1999.

[27] B. Y. Alabyev, A. M. Najakshin, L. V. Mechetina, and A. V. Taranin, "Cloning of a CXCR4 homolog in chondrostean fish and characterization of the CXCR4-specific structural features," Developmental and Comparative Immunology, vol. 24, no. 8, pp. 765-770, 2000.

[28] E. Chaves-Pozo, P. Muñoz, A. López-Muñoz, et al., "Early innate immune response and redistribution of inflammatory cells in the bony fish gilthead seabream experimentally infected with Vibrio anguillarum," Cell and Tissue Research, vol. 320, no. 1, pp. 61-68, 2005.

[29] W. Zheng, C. Tian, and X. Chen, "Molecular characterization of goose-type lysozyme homologue of large yellow croaker and its involvement in immune response induced by trivalent bacterial vaccine as an acute-phase protein," Immunology Letters, vol. 113, no. 2, pp. 107-116, 2007.

[30] B. Novoa, T. V. Bowman, L. Zon, and A. Figueras, "LPS response and tolerance in the zebrafish (Danio rerio)," Fish and Shellfish Immunology, vol. 26, no. 2, pp. 326-331, 2009.

[31] L. M. Sly, M. J. Rauh, J. Kalesnikoff, C. H. Song, and G. Krystal, "LPS-induced upregulation of SHIP is essential for endotoxin tolerance," Immunity, vol. 21, no. 2, pp. 227-239, 2004.

[32] H. Shim, S. K. Lau, S. Devi, Y. Yoon, H. T. Cho, and Z. Liang, "Lower expression of CXCR4 in lymph node metastases than in primary breast cancers: potential regulation by ligand-dependent degradation and HIF-1 $\alpha$," Biochemical and Biophysical Research Communications, vol. 346, no. 1, pp. 252-258, 2006.

[33] A. Faber, C. Roderburg, F. Wein, et al., "The many facets of SDF- $1 \alpha$, CXCR 4 agonists and antagonists on hematopoietic progenitor cells," Journal of Biomedicine and Biotechnology, vol. 2007, no. 3, p. 26065, 2007.

[34] M. C. Ordás, M. M. Costa, F. J. Roca, et al., "Turbot TNF $\alpha$ gene: molecular characterization and biological activity of the recombinant protein," Molecular Immunology, vol. 44, no. 4, pp. 389-400, 2007.

[35] M. L. Rise, J. Hall, M. Rise, et al., "Functional genomic analysis of the response of Atlantic cod (Gadus morhua) spleen to the viral mimic polyriboinosinic polyribocytidylic acid (pIC)," Developmental and Comparative Immunology, vol. 32, no. 8, pp. 916-931, 2008.

[36] S. Varani, G. Frascaroli, M. Homman-Loudiyi, S. Feld, M. P. Landini, and C. Söderberg-Nauclér, "Human cytomegalovirus inhibits the migration of immature dendritic cells by downregulating cell-surface CCR1 and CCR5," Journal of Leukocyte Biology, vol. 77, no. 2, pp. 219-228, 2005.

[37] J. Montero, A. Estepa, J. Coll, and C. Tafalla, "Regulation of rainbow trout (Oncorhynchus mykiss) interleukin-8 receptor (IL-8R) gene transcription in response to viral hemorrhagic septicemia virus (VHSV), DNA vaccination and chemokines," Fish and Shellfish Immunology, vol. 25, no. 3, pp. 271-280, 2008.

[38] J. L. Pablos, A. Amara, A. Bouloc, et al., "Stromal-cell derived factor is expressed by dendritic cells and endothelium in human skin," American Journal of Pathology, vol. 155, no. 5, pp. 1577-1586, 1999.

[39] A. Müller, B. Homey, H. Soto, et al., "Involvement of chemokine receptors in breast cancer metastasis," Nature, vol. 410, no. 6824, pp. 50-56, 2001. 
[40] E. Palmesino, B. Moepps, P. Gierschik, and M. Thelen, "Differences in CXCR4-mediated signaling in B cells," Immunobiology, vol. 211, no. 5, pp. 377-389, 2006.

[41] C. Dambly-Chaudière, N. Cubedo, and A. Ghysen, "Control of cell migration in the development of the posterior lateral line: antagonistic interactions between the chemokine receptors CXCR4 and CXCR7/RDC1," BMC Developmental Biology, vol.

7, article 23, 2007. 


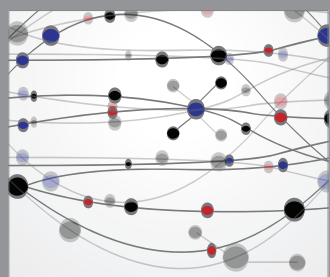

The Scientific World Journal
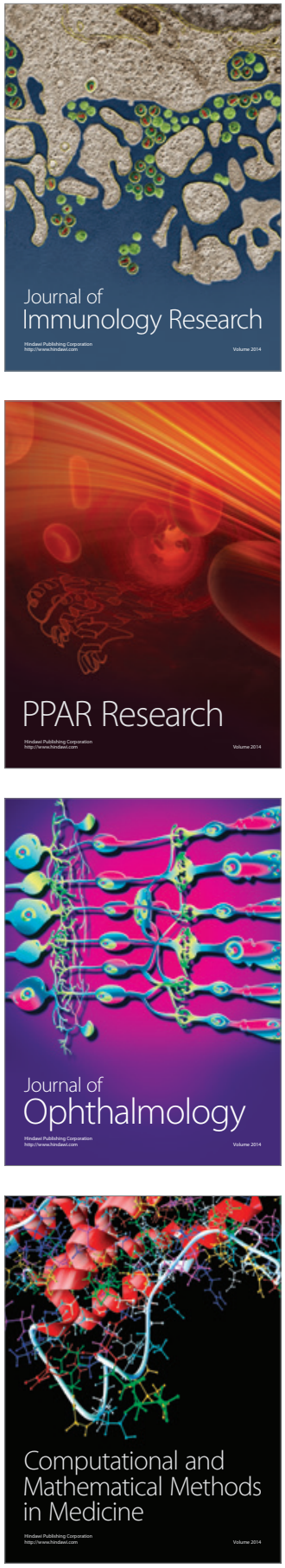

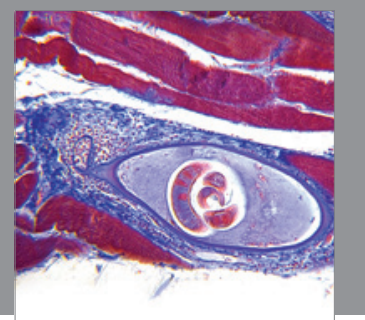

Gastroenterology

Research and Practice
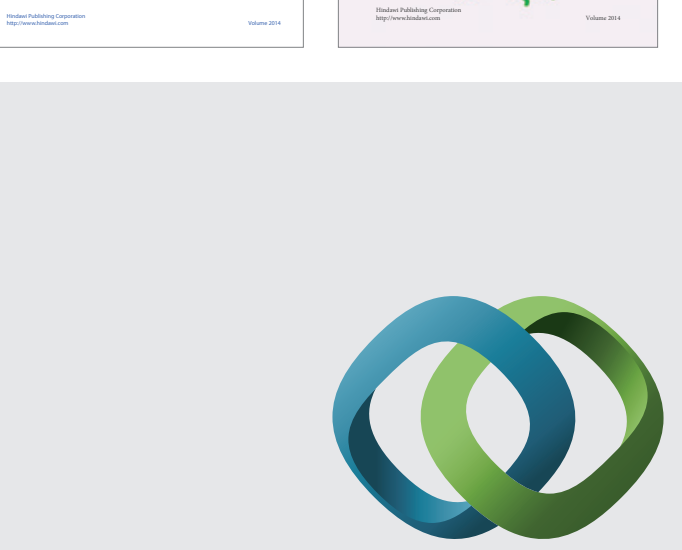

\section{Hindawi}

Submit your manuscripts at

http://www.hindawi.com
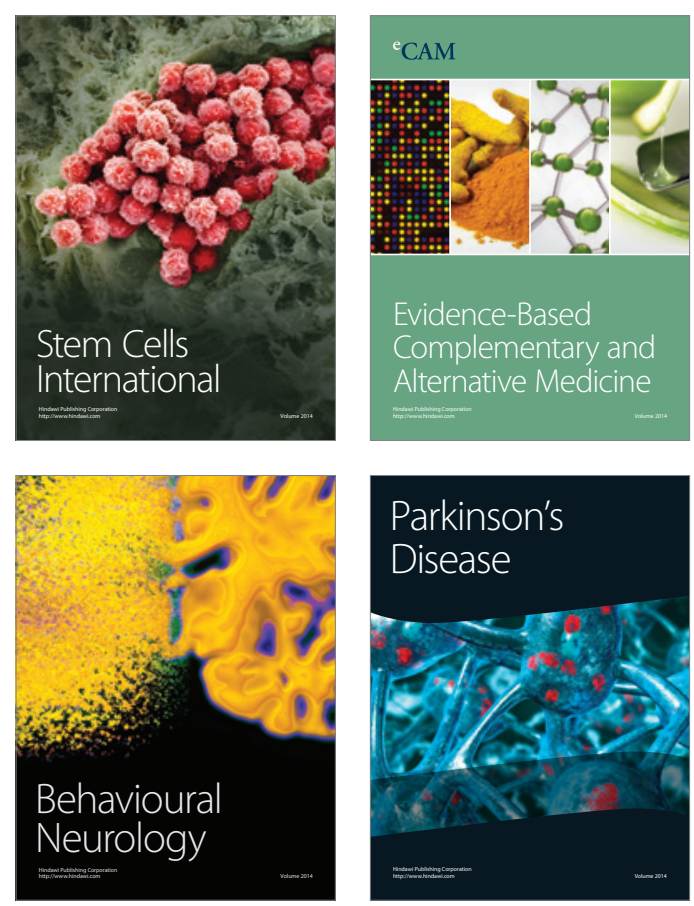

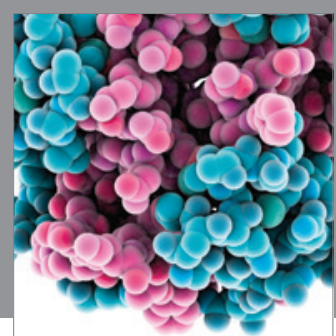

Journal of
Diabetes Research

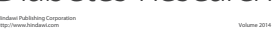

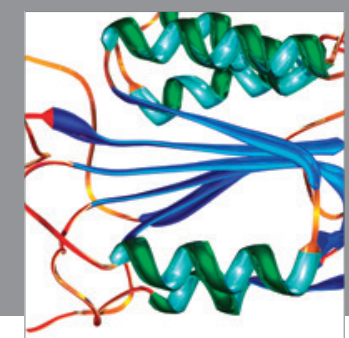

Disease Markers
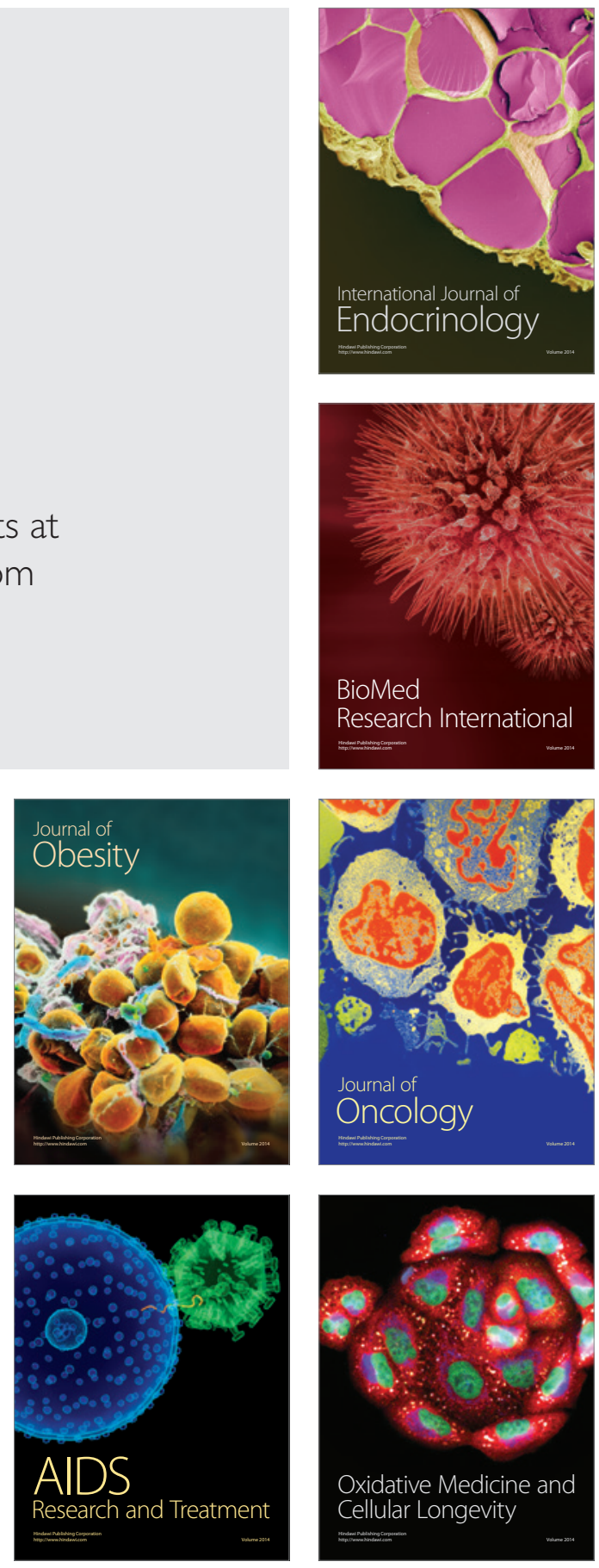